\title{
Network Resource Model for 5G Network and Network Slice
}

\author{
Jing Ping \\ Rapporteur of 3GPP SA5, Nokia, China \\ E-mail: jing.ping@nokia-sbell.com \\ Received 27 February 2019; \\ Accepted 20 May 2019
}

\begin{abstract}
To support management and orchestration of 5G networks, the Network Resource Model (NRM) is an information model representing the manageable aspects of $5 \mathrm{G}$ networks. The NRM needs to be defined, according to $5 \mathrm{G}$ network specifications from other $3 \mathrm{GPP}$ working groups as well as considering requirements for the $5 \mathrm{G}$ management architecture and operations.

A Network Resource is a component which can be identified as a discrete entity and is in an object-oriented environment for the purpose of management represented by an abstract entity called Information Object Class (IOC).

IOCs, attributes of an IOC and relationships between IOCs are defined in the NRM.

This paper describes how the 5G NRM is specified in 3GPP in three stages including requirements, information service and solution set definitions.
\end{abstract}

Keywords: NRM, UML, XML, JSON, YANG, IOC.

\section{Introduction}

The 3GPP network management architecture has evolved to a service-based management framework in Release 15, wherein a Management Service (MnS) combines elements of management service components type A, B and C.

Journal of ICT, Vol. 7_2, 127-140. River Publishers

doi: 10.13052/jicts2245-800X.724

This is an Open Access publication. (c) 2019 the Author(s). All rights reserved. 
The MnS components are combined to allow an MnS consumer to interact with an MnS producer via a specified service interface. The NRM specifies $\mathrm{MnS}$ components of type $\mathrm{B}$, which is the management information represented by an information model of managed entities.

Figure 4.3.1 in 3GPP TS 28.533 [8], also included in the article named "5G Management and Orchestration Framework" in this Journal issue, shows examples of how these three component types can be used to compose different management services.

An MnS component type A provides services to manage the lifecycle of IOC instances (a.k.a Managed Object Instances, MOIs, or simply managed objects), and set/get attributes of the MOI, as well as provide performance assurance and fault supervision for the MOI. An MnS component type C comprises performance information or fault/alarm information related to a measured or monitored MOI. A Distinguish Name (DN) is used to link the performance or alarm data to the MOI.

The Scope of the 3GPP 5G NRM is described in Section 2. Section 3 introduces methodology used for 3GPP NRM definition. High level 3GPP NRM stage 1, 2 and 3 definitions are given in Sections 4-6. Finally, the paper concludes the 3GPP 5G NRM achievement and future work in Section 7.

\section{Scope of 5G NRM}

As shown in Figure 1, vertically, the 5G NRM supports modelling of NGRAN, 5G Core and Network Slice, as well as a Generic NRM (in TS 28.622 [2]) which can be reused or inherited by other domain specific models.

Horizontally, the NRM provides Stage 1, Stage 2 and Stage 3 definitions for generic and domain specific managed objects. The different stages have the following meaning:

\section{Stage 1:}

The "Requirements-level" intends to provide conceptual and use cases definitions for a specific network resource as well as defining subsequent requirements for this resource.

\section{Stage 2:}

The "Information Service (IS)-level" provides the technology independent specification of a network resource. 


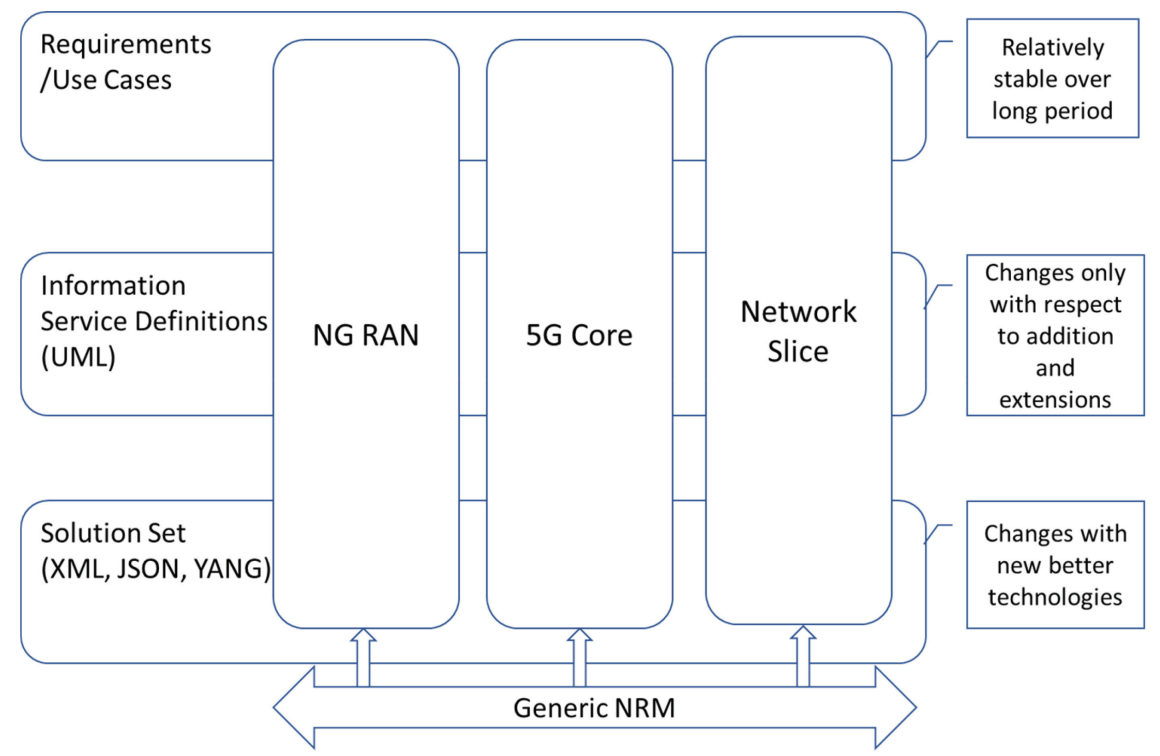

Figure 1 Scope of the 3GPP NRM for 5G network.

\section{Stage 3:}

The "Solution Set (SS)-level" finally provides the mapping of IS definitions into one or more technology-specific Solution Sets. This concept provides support for multiple technologies as applicable on network type basis and also enables accommodation of future technologies - without the need to redefine requirements and IS-level definitions.

This paper is organized based on the stages of NRM definitions.

\section{Methodology of 5G NRM Definition}

\subsection{Design Principles}

- Separation of Stage 2 and Stage 3, where Stage 2 specifies the semantics exchanged between network management consumers and producers and Stage 3 specifies the syntax of the semantics exchanged. This allows to define new Stage 3 Solution Sets for new technologies without changing the Stage 2 Information Service.

- Protocol technology independent Stage 3 definition, which decouples the NRM from the communication protocols. For example, an XML Solution Set can be used to support both Web Service, NetConf or other 
communication protocols, and a YANG Solution Set is not necessarily bound to the NetConf protocol.

- Decoupling of management service components type A, B and C, which allows to define IOC agnostic generic management service. E.g. the CreateMOI operation of the generic provisioning service can be applied on either NG-RAN, 5GC or Network Slice IOCs without definition change.

- Umbrella Information Model (UIM) definition (in TS 28.620 [1]), which is necessary for the harmonization of various Domain/Technologyspecific model classes (for example, wire-line and wireless networks), forms the so-called Federated Network Information Model (FNIM).

\subsection{Stage 2 Definition Guidelines}

To standardize and formalize NRM definitions, an NRM Stage 2 Technical Specification template and related guidelines are defined in TS 32.160 [7] in Release 16, including naming convention and constraints of each component defined in Stage 2, etc.

\subsection{Stage 3 Definition Guidelines}

3GPP TS 32.160 [7] also defines templates and guidelines for Stage 3 TSs. Besides solution specific guidelines defined for each Solution Set, some convergence style guides provide design rules for mapping Stage 2 to Stage 3 items in a consistent way to make XML, YANG, JSON and other stage 3 definitions look similar. This includes the following:

- Managed Object Identifier represented in stage 3

- Managed Object Class represented in stage 3

- Managed Object Attributes represented in stage 3

- Naming containment represented in stage 3

- Module Structure of stage 3

- etc.

\section{NRM Stage 1}

The 5G NRM Stage 1 specification defines requirements including:

- Generic requirements for management of entities including converged network and virtualized network functions as enabler of $5 \mathrm{G}$. 
- Requirements for management of NG Radio Access Network (NG-RAN; the new RAN for 5G) including Multi-RAT Dual Connectivity (MR-DC) support.

- Requirements for management of 5G Core (5GC) Network Function including edge computing and AMF load balancing support.

- Requirements for management of Network Slice and Network Slice Subnet.

All 5G NRM related requirements are defined in TS 28.540 [4].

\section{NRM Stage 2}

The NRM Stage 2 specification defines the semantics and behavior of Information Object Classes (IOCs), attributes of IOCs, and relations visible on the management interfaces in a protocol and technology neutral way. The NRM is structured according to Generic NRM, NG-RAN, 5GC, and Network slice definitions.

\subsection{Generic NRM}

As shown in Figure 2, the Generic NRM defined in TS 28.622 [2] specifies the generic network resource information which mainly includes root and abstract IOCs, such as SubNetwork, ManagedElement and ManagedFunction. To support Fixed-Mobile Convergence (FMC) use cases and requirements, the 3GPP NRM supports the Federated Network Information Model (FNIM)

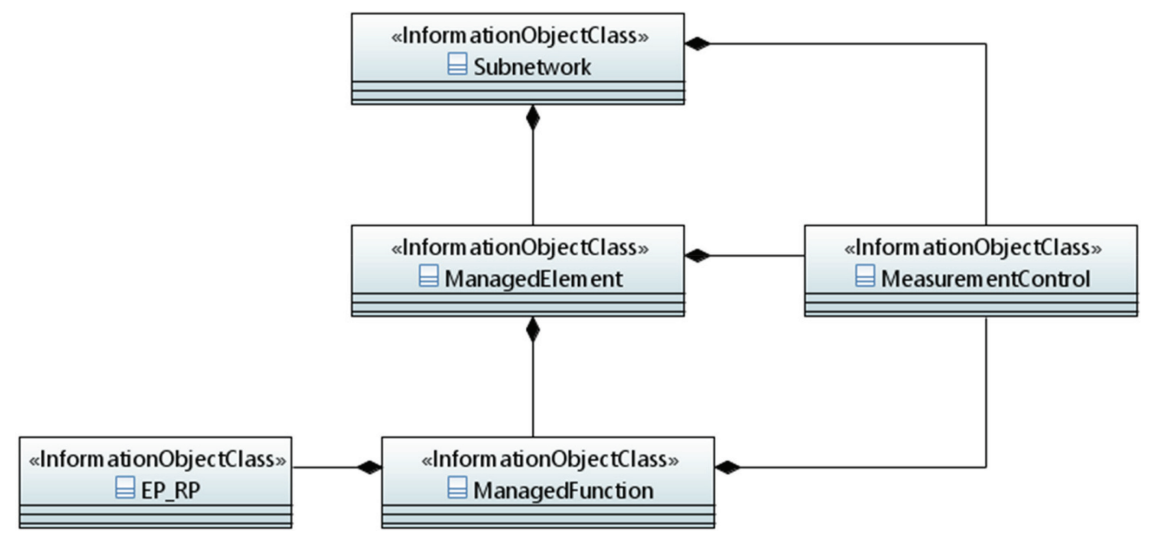

Figure 2 Generic NRM Class Diagram of Release 15. 
concept in that the relevant IOCs defined in the Generic NRM directly or indirectly inherited from those specified in the Umbrella Information Model defined in TS 28.620 [1].

To better support 5G network management, a service-based management framework has been introduced by 3GPP SA5 since Release 15. Accordingly, NRM based performance measurements control was proposed to align with the service-based management framework. It allows the performance measurements control being configurable through generic provisioning services such as create IOC, get/modify IOC attributes, etc. The NRM is extended to add MeasurementControl IOC definition which represents the capabilities to produce and deliver measurements of relevant managed objects.

Similarly, Trace control related classes could be defined in the NRM for Trace Session Control in Release 16.

\subsection{NG-RAN}

In the NG-RAN NRM defined in TS 28.541 [5], a corresponding IOC is defined for each network function of gNB (the 5G base station) in the so-called 3-split deployment scenarios with CU-CP (Control Plane), CU-UP (User Plane) and DU (see TS 38.401 [12]). Figure 3 shows the IOCs and containment relationship between high-level and radio cell-related IOCs.

The NRM also defines the attributes modelling the properties of each IOC, e.g. related to the gNB device configuration, cell configuration, neighbor cell configuration, frequency configuration, network slice configuration and policy configuration for efficient resource sharing between slices.

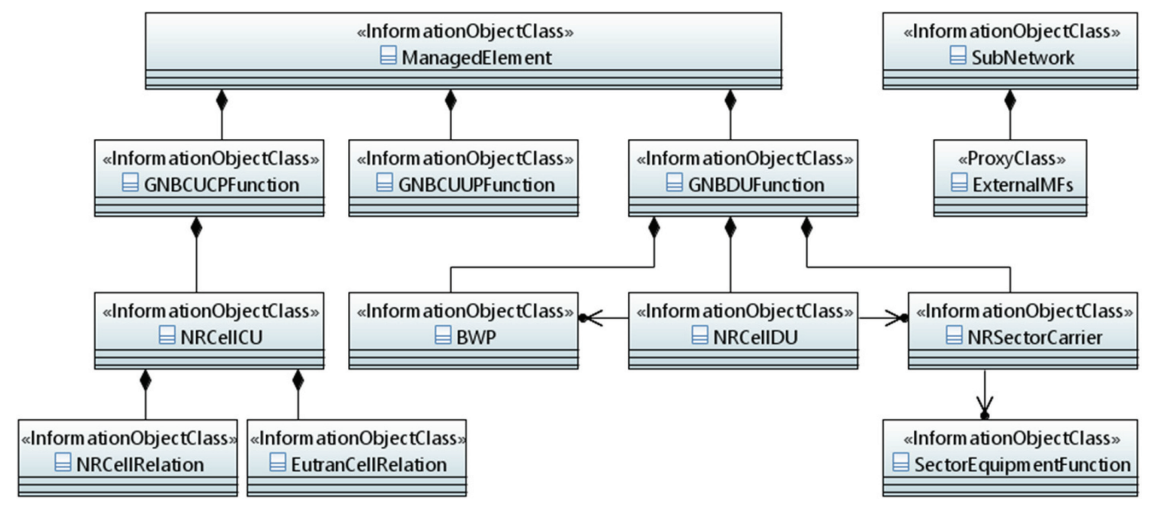

Figure 3 NG-RAN Class Diagram. 


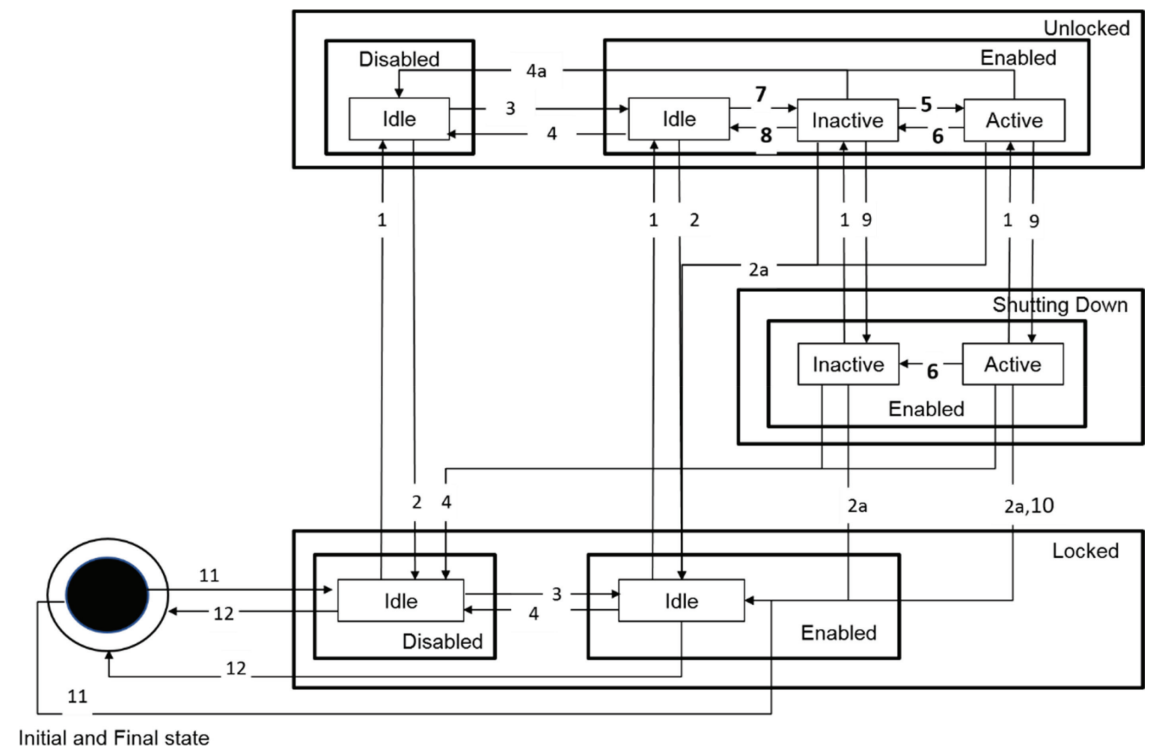

Figure 4 Combined gNB cell state diagram.

In addition, the interaction between gNB Network Functions (NFs), and between gNB NF and Core or eNB NF, is modelled by the EP_RP IOC which represents an Endpoint (EP) of a link used across a reference point between two network entities.

The Transport view of the (4G) eUTRAN in TS 28.658 [11] has also been updated accordingly to reflect the interaction between the $4 \mathrm{G}$ eNB and $5 \mathrm{G}$ gNB.

States and state machine of a cell is also defined in the NG-RAN NRM, as shown in Figure 4.

\section{$5.35 G C$}

This clause comprises the set of classes (e.g. IOCs) that encapsulates the information relevant for the NRM of 5GC NFs. Figure 5 shows a compressed high-level view of the 5GC NF NRM containment/naming relationships.

The InternalCoreMFs ProxyClass represents all Managed Functions of the 5G Core Network Functions managed by a specific Management Function. The NFs include AMF, SMF, UPF, PCF, NRF, NSSF, AUSF, UDM, LMF, 5GEIR, UDR, SMSF, UDR, etc. 

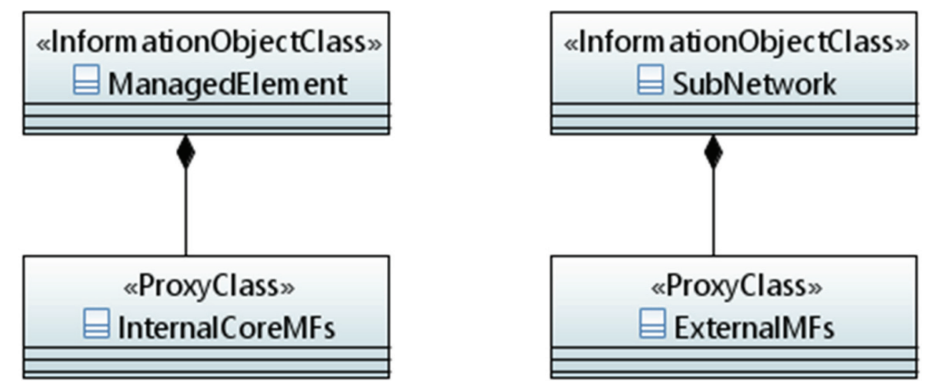

Figure 5 5GC Class Diagram.

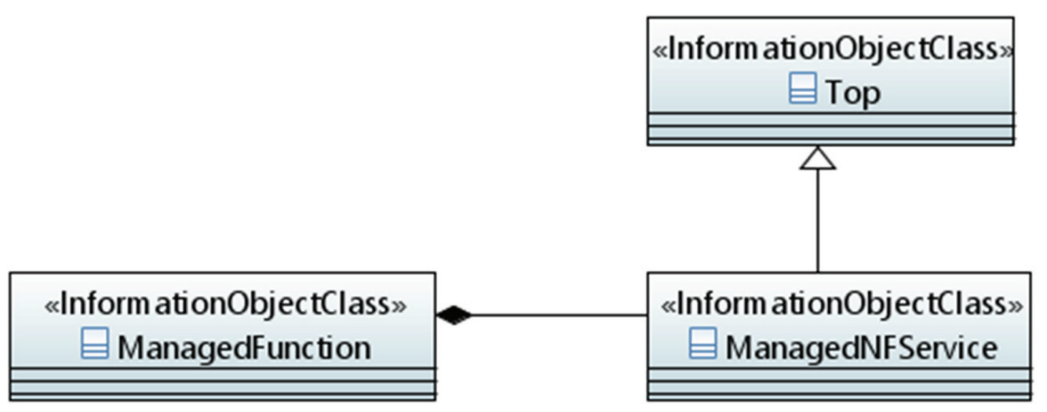

Figure 6 NRM segment to support 5G Core NF Service.

The ExternalMFs ProxyClass represents all Managed Functions of the 5G Core or Radio Network Functions known by a specific Management Function but managed by another Management Function. The NFs can be 5G Core NFs list above, or RAN NFs such as gNBCUCP, gNBCUUP, etc.

The reference point based 5G Core architecture requires a large number of EPs to be defined and configured by the management function. Those EPs and their attributes are specified in TS 28.541 [5]. In addition, a new Release 16 work item has been started in SA5 to enhance the NRM to support the Service Based Architecture of 5G Core. ManagedNFService IOC is introduced to represent a Network Function (NF) Service as defined in 3GPP TS 23.501 [13]. Figure 6 shows containment and inheritance relationship between the ManagedNFService IOC and other existing NRM IOCs (refer to 3GPP TS 28.622 [2]). 


\subsection{Network Slice}

To support management and orchestration of Network Slice and Network Slice Subnet (NSS), which represents the management aspects of a set of Managed Functions and the required resources (e.g. compute, storage and networking resources), an information model fragment for Network Slice and NSS is defined in the 5G NRM. Both IOCs inherit from Subnetwork. Figure 7 shows Network Slice related classes and association/aggregation relationships. The Network Service and VNF classes are defined by ETSI NFV specifications.

In addition, the 5G NRM defines states and state machine for the Network Slice and NSSI as shown in Figure 8.

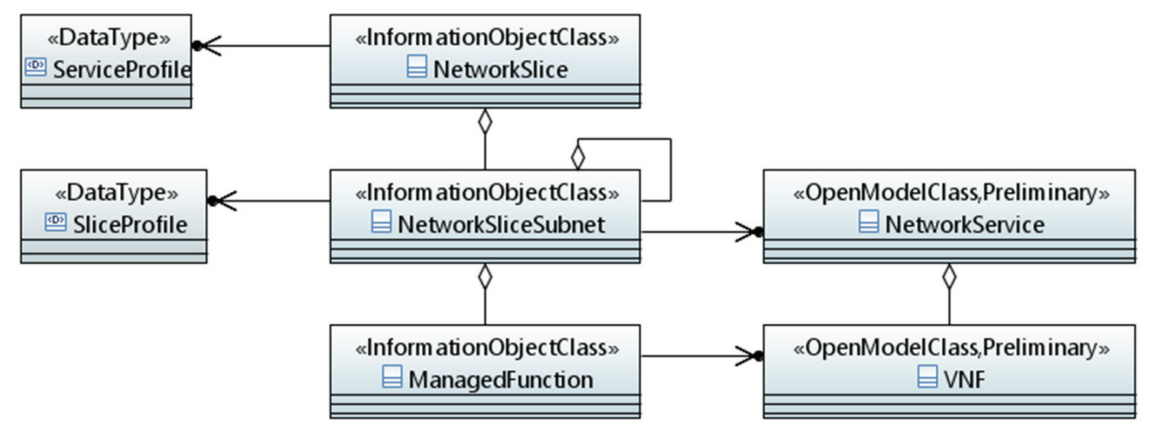

Figure 7 Network Slice Class Diagram.

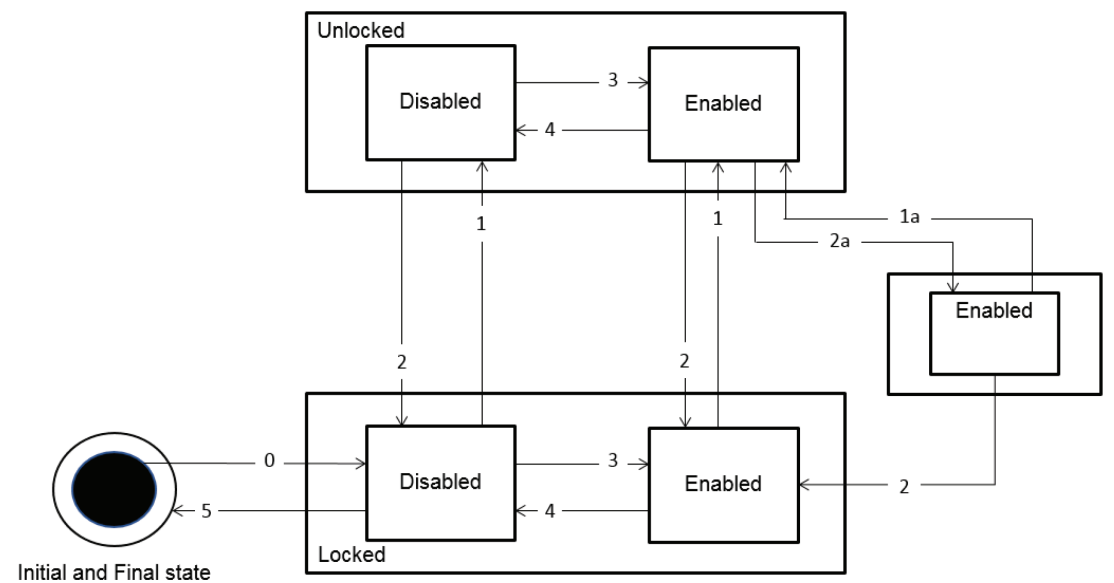

Figure 8 Combined NSI/NSSI state diagram. 


\section{NRM Stage 3}

NRM Stage 3 definitions map the Stage 2 Information Service definitions to a specific Solution Set definition used for implementations. NRM Stage 3 definitions for 5G including XML, JSON and YANG Solution Set are found in Annexes of TS 28.541 [5]. With emerging new technologies or industry requirements, additional Solution Sets can be supported without changing the Stage 2 NRM definitions.

\subsection{XML}

The XML solution set is mainly used for Bulk Configuration Management (CM) specified in TS 32.611 [14] and TS 32.612 [15].

NRM-specific XML schemas for Bulk CM, corresponding 3GPP TSs and XML namespace prefixes, and generic mapping rules for NRM-specific XML element types are defined in TS 32.616 [10].

Generic XML NRM Solution Set in TS 28.623 [3] and XML-based 3GPP NG-RAN, 5GC and Network Slice NRM Solution Set are defined in TS 28.541 [5] to support the complete management of 5G networks including Network Slices.

The following XML Schema are used for 5G network management.

- "http://www.3gpp.org/ftp/specs/archive/28_series/28.623\#genericNrm"

- "http://www.3gpp.org/ftp/specs/archive/28_series/28.626\#state ManagementIRP"

- "http://www.3gpp.org/ftp/specs/archive/28_series/28.659\#eutranNrm"

- "http://www.3gpp.org/ftp/specs/archive/28_series/28.541\#nrNrm"

- "http://www.3gpp.org/ftp/specs/archive/28_series/28.541\#ngcNrm"

- "http://www.3gpp.org/ftp/specs/archive/28_series/28.541\#sliceNrm"

\subsection{JSON}

The JSON Solution Set is introduced to mainly support RESTful HTTPbased solutions, especially to adapt to the service-based management framework.

JSON object and type definition guidelines are specified in TS 32.158 [6].

The Generic JSON NRM Solution Set is defined in TS 28.623 [3], and JSON based 3GPP NG-RAN, 5GC and Network Slice NRM Solution Set are defined in TS 28.541 [5] to support the complete management of 5G networks including Network Slices. 
The following JSON Schema are used for 5G network management.

- "http://3gpp.org/28623/genericNrm.json"

- "http://3gpp.org/28626/stateManagementNrm.json"

- "http://3gpp.org/28541/nrNrm.json"

- "http://3gpp.org/28541/ngcNrm.json"

- "http://3gpp.org/28541/sliceNrm.json"

\subsection{YANG}

The YANG Solution Set is defined in 3GPP for being reused by Open Source Projects such as ONAP, O-RAN, especially for node configuration.

The general rules for YANG modulization and UML to YANG mapping are defined in TS 32.160 [7].

Generic YANG NRM Solution Set, YANG based 3GPP NG-RAN, 5GC and Network Slice NRM Solution Set are defined in TS 28.541 [5] to support the complete management of $5 \mathrm{G}$ networks including Network Slices.

The following YANG modules including related sub-modules are defined for $5 \mathrm{G}$ network management.

- General type definition "nrm-types-3gpp.yang"

- Basic IOC definition "Top.yang", "ManagedElement.yang", "ManagedFunction.yang", "EP_RP.yang", "Subnetwork.yang", "VsDataContainer.yang", "MeContext.yang", "ManagementNode.yang"

- NGRAN, composition of all gNB ManagedFunctions in "ngran.yang"

- NGC module, composition of all 5GC ManagedFunctions in "ngc.yang"

- NetworkSlice Module, "NetworkSlice.yang"

- NetworkSlice Module, "NetworkSliceSubnet.yang"

\section{Conclusion and Outlook}

The 5G NRM defined in 3GPP SA5 Release 15 enables 5G network and network slice management automation, enables multi-vendor interoperability, and facilitates interaction between different SDOs, as well as between SDOs and Open Source Projects.

Methodologies applied in the 3GPP SA5 NRM definition, including separation of Stage 2 and Stage 3, IOC independent operations, protocol independent Stage 3, and an Umbrella Information Model definition, make the 5G NRM extendable and flexible, and allow it to synergize with other SDOs and Open Source Projects. 
Most features and definitions of the 5G NRM described in this paper are referring to 3GPP Release 15 specifications. In Release 16 and beyond, the following technical or non-technical enhancements are under development:

- Full support of the 5G Core Service Based Architecture (SBA)

- NRM based trace management to align with service-based management framework

- Support more configuration attributes for NG-RAN, 5G Core and Network Slice

In addition, we are defining specifications to maximize the automation of network management, and the same principle is expected to be adopted in our daily standardization work for specification development, review, and maintenance, hence we will leverage open source and tools to:

- Manage Stage 3 change tracking and review

- Utilize agile processes used by open source projects and IT industry associations

\section{References}

[1] 3GPP TS 28.620: "Telecommunication management; Fixed Mobile Convergence (FMC) Federated Network Information Model (FNIM) Umbrella Information Model (UIM)", V15.0.0.

[2] 3GPP TS 28.622: "Telecommunication management; Generic Network Resource Model (NRM) Integration Reference Point (IRP); Information Service (IS)", V15.2.0.

[3] 3GPP TS 28.623: "Telecommunication management; Generic Network Resource Model (NRM) Integration Reference Point (IRP); Solution Set", V15.1.0.

[4] 3GPP TS 28.540 Management and orchestration; 5G Network Resource Model (NRM); Stage 1, V15.1.0.

[5] 3GPP TS 28.541 Management and orchestration; 5G Network Resource Model (NRM); Stage 2 and Stage 3, V15.1.0.

[6] 3GPP TS 32.158: "Management and orchestration; Design rules for REpresentational State Transfer (REST) Solution Sets (SS)", V15.1.0.

[7] 3GPP TS 32.160 Management and orchestration; Management service template, V1.0.1.

[8] 3GPP TS 28.533 "Management and orchestration; Architecture framework", V15.1.0. 
[9] 3GPP TS 32.150 "Telecommunication management; Integration Reference Point (IRP) Concept and Definitions", V15.0.0.

[10] 3GPPTS 32.616 "Telecommunication management; Configuration Management (CM); Bulk CM Integration Reference Point (IRP) Solution Sets (SS)", V15.0.0.

[11] 3GPP TS 28.658 "Telecommunications management; Evolved Universal Terrestrial Radio Access Network (E-UTRAN) Network Resource Model (NRM) Integration Reference Point (IRP): Information Service (IS)", V15.2.0.

[12] 3GPP TS 38.401 "NG-RAN; Architecture description", V15.2.0.

[13] 3GPP TS 23.501 "System Architecture for the 5G System", V15.3.0.

[14] 3GPPTS 32.611 "Telecommunication management; Configuration Management (CM); Bulk CM Integration Reference Point (IRP) Requirements", V15.0.0.

[15] 3GPPTS 32.612 "Telecommunication management; Configuration Management (CM); Bulk CM Integration Reference Point (IRP) Information Service (IS)", V15.0.0.

\section{Biography}

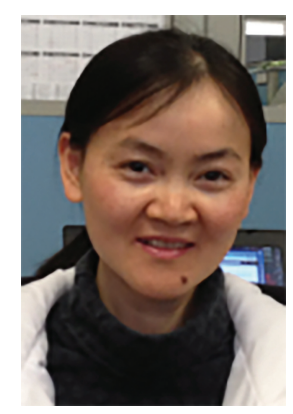

Jing Ping is a senior standardization specialist at Nokia Bell Labs, in China, where she is currently involved in standardization and research activities spanning different areas such as network management and security automation. She received computer science bachelor degree from University of Electronic Science and Technology of China, and joined Motorola, then Nokia in 1999.

She has been working as a product manager, system and software architect and software engineer in mobile core network, network management, cloud and security management, and information security domains. 
